\title{
onkopedia
}

onkopedia guidelines

\section{Chronic Lymphocytic Leukemia (CLL)}

\section{Guideline}

Recommendations from the society for diagnosis and therapy of haematological and oncological diseases 


\section{Publisher}

DGHO Deutsche Gesellschaft für Hämatologie und Medizinische Onkologie e.V.

Alexanderplatz 1

D-10178 Berlin

Executive chairman: Prof. Dr. med. Carsten Bokemeyer

Phone: $+49(0) 3027876089$ - 0

Fax: +49(0)3027876089- 18

info@dgho.de

www.dgho.de

\section{Contact person}

Prof. Dr. med. Bernhard Wörmann

Medical superintendent

\section{Source}

www.onkopedia-guidelines.info

The information of the DGHO Onkopedia Web Site is not intended or implied to be a substitute for professional medical advice or medical care. The advice of a medical professional should always be sought prior to commencing any form of medical treatment. To this end, all component information contained within the web site is done so for solely educational purposes. DGHO Deutsche Gesellschaft für Hämatologie und Onkologie and all of its staff, agents and members disclaim any and all warranties and representations with regards to the information contained on the DGHO Web Site. This includes any implied warranties and conditions that may be derived from the aforementioned web site information. 


\section{Table of contents}

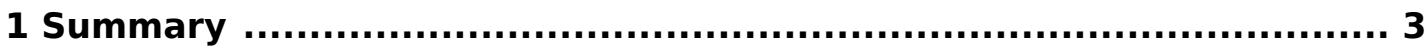

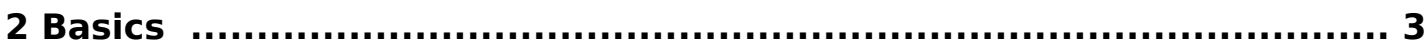

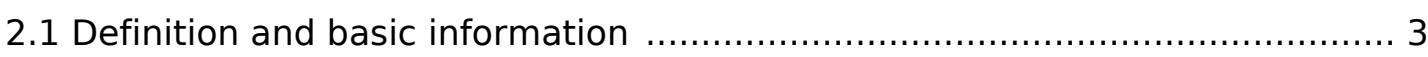

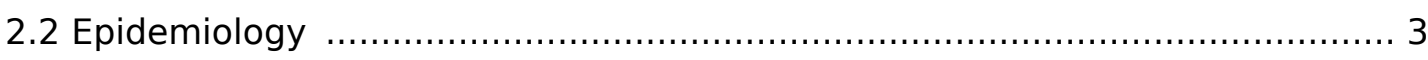

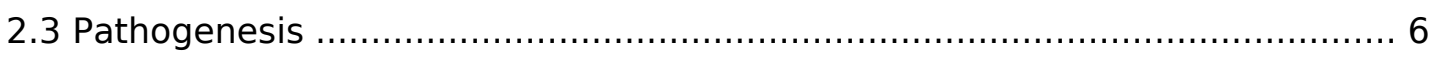

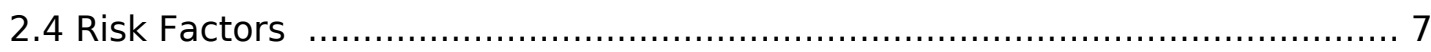

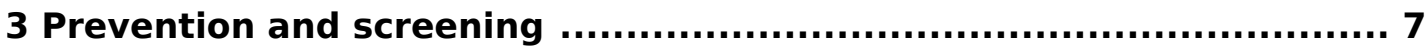

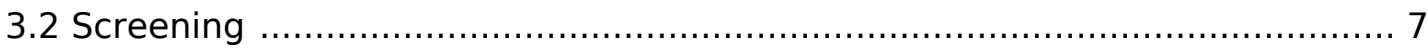

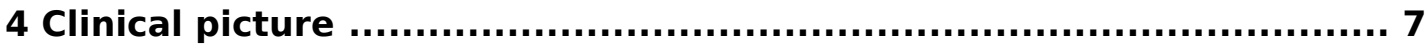

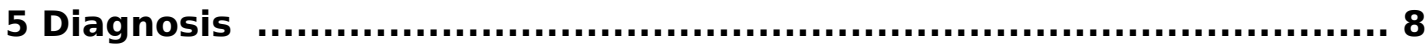

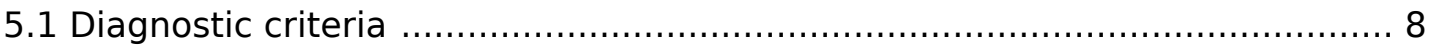

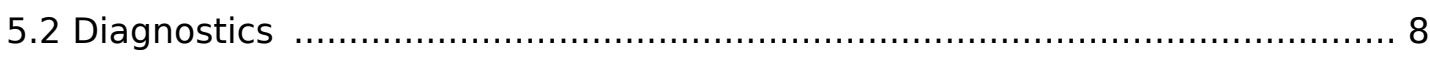

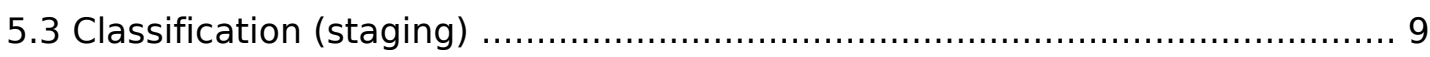

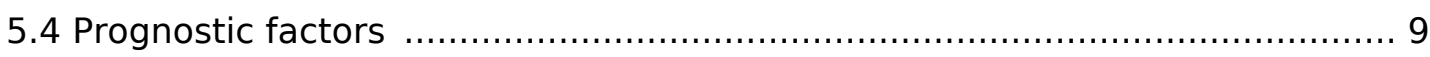

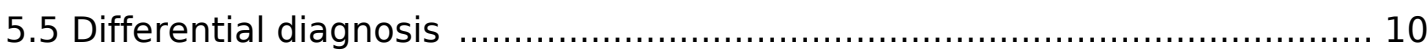

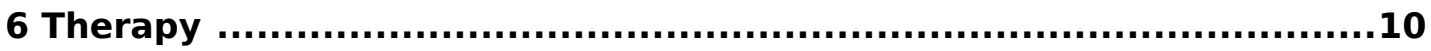

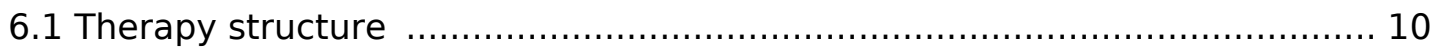

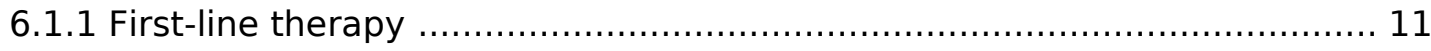

6.1.1.1 Patients without del(17p13) or TP53 mutation ............................... 12

6.1.1.2 Patients with a confirmed del(17p13) or TP53 mutation .................... 13

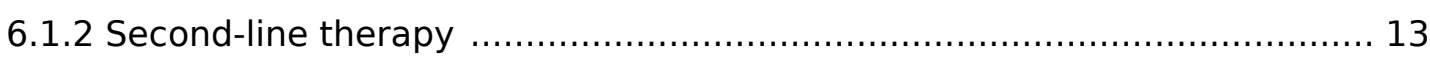

6.1.2.1 Progressive disease / therapy resistance / early relapse .................... 14

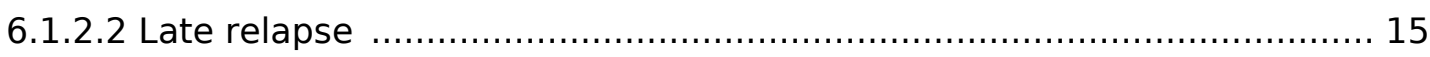

6.1.3 Allogeneic stem cell transplantation .................................................. 15

6.1.4 Autologous stem cell transplantation .......................................... 15

6.1.5 Supportive therapy and treatment of complications ............................ 16

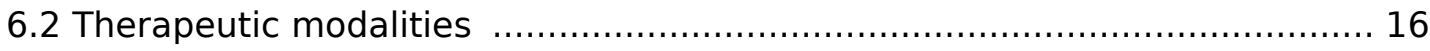

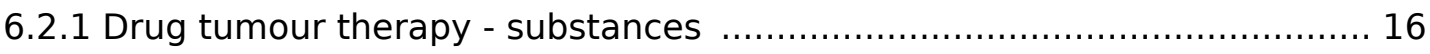

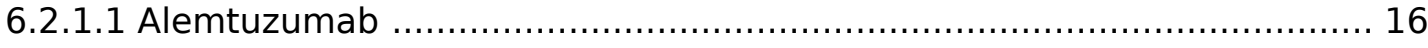

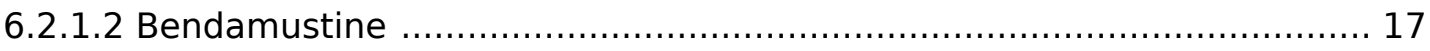

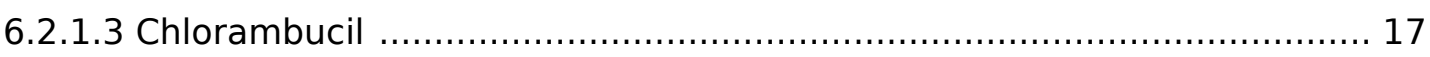

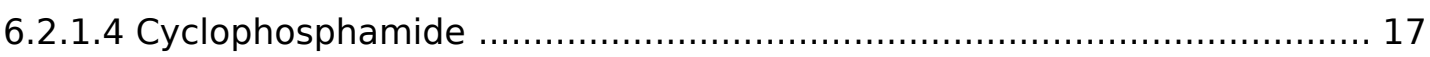

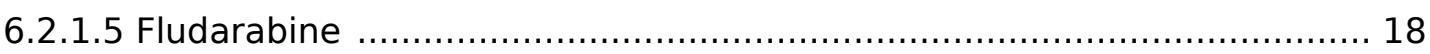

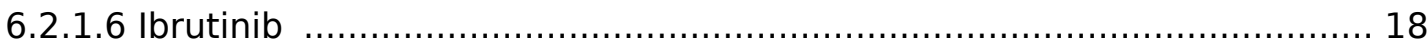




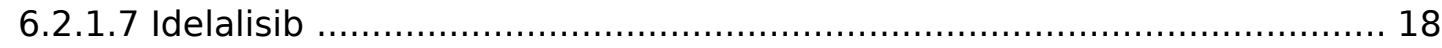

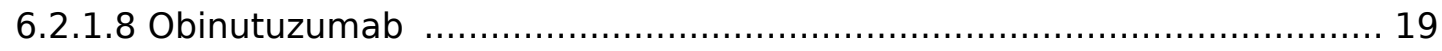

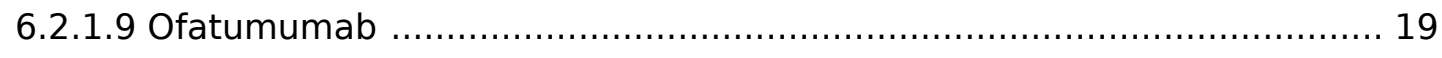

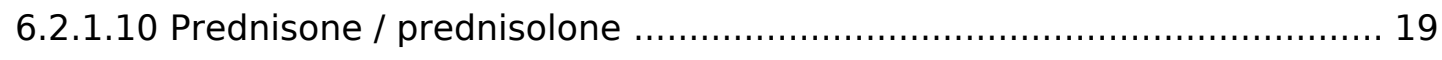

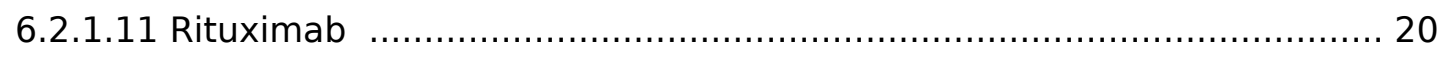

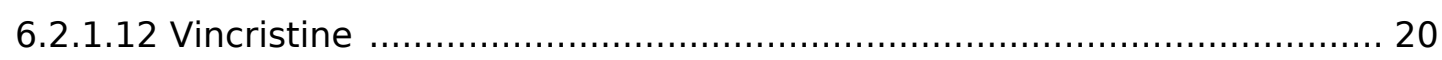

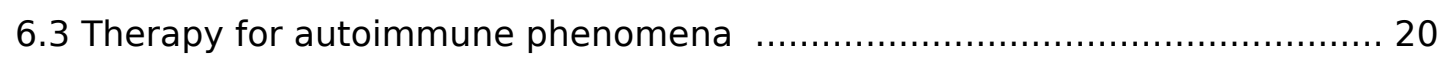

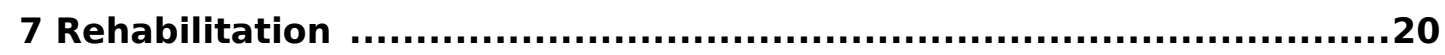

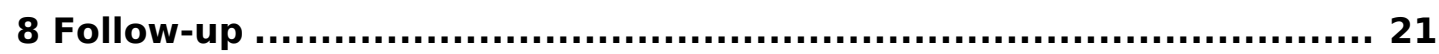

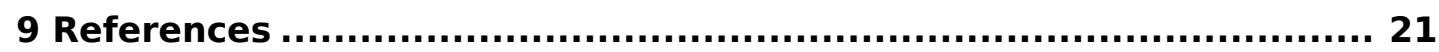

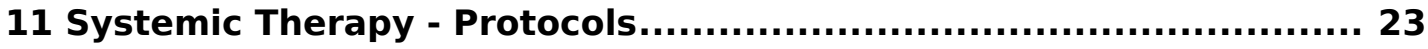

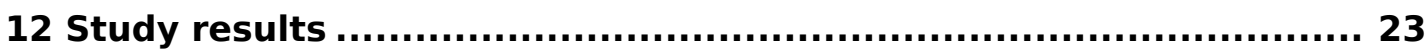

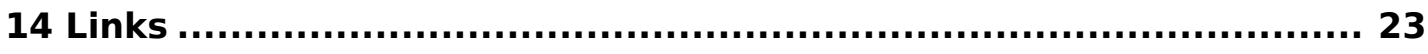

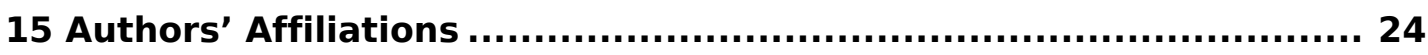




\section{Chronic Lymphocytic Leukemia (CLL)}

Status: November 2014

Authors: Clemens-Martin Wendtner, Peter Dreger, Michael Gregor, Richard Greil, Wolfgang Ulrich Knauf, Ron Pritzkuleit, Johannes Schetelig, Michael Steurer, Stephan Stilgenbauer

\section{Summary}

Chronic lymphocytic leukemia (CLL) is the most common leukemic disease in Central Europe. CLL is clinically and biologically heterogeneous. The median age of onset is between 70 and 75 years, but with a large age span. The disease is preceded by monoclonal $B$ lymphocytosis (MBL).

Antineoplastic treatment is initiated only in symptomatic patients. The choice of first-line therapy depends on the general condition of the patient and any relevant comorbidities. The combination of chemotherapy with monoclonal anti-CD20 antibodies has improved the prognosis of CLL patients. Kinase inhibitors are a new class of effective drugs which interfere with signal transduction via the B-cell receptor.

\section{Basics}

\subsection{Definition and basic information}

The WHO classification describes Chronic Lymphocytic Leukemia (CLL) as an indolent (lymphocytic) lymphoma, characterised by a leukemic course. According to the WHO, CLL is always a B-cell neoplasm, while the condition formerly designated as T-CLL is now referred to as T-prolymphocytic leukemia (T-PLL).

\subsection{Epidemiology}

CLL is the most common leukemic disorder encountered in Western industrialised nations. In Germany about 3,000 men and 2,000 women become are newly diagnosed with this disease each year (see Figure 1 ). This corresponds in men to a proportion of about $1.1 \%$ of all cancers (excluding skin cancers that are not melanomas) and about $41.8 \%$ of all leukemia cases. In women, both proportions are slightly lower. CLL accounts for $0.8 \%$ of cancer overall and $37.2 \%$ of all leukemia cases [1].

The cumulative incidence (number of new cases) in 2011 was 7.4 per 100,000 in men and 4.8 per 100,000 in women. Over the past nine years the age-standardised incidence revealed no uniform trend (see Figure 2). With only slight variations, the incidences for both sexes have remained for the most part constant.

People with an African or Asian / Pacific genetic background have a lower risk of developing the disease than whites [2]. 
CLL is a disease of senior age, see Figure 3 and Figure 4. The average (median) age of onset in 2011 was 72 years for men and 75 years for women. For men the highest age-specific cumulative incidence is in the age group of 80 to 84 years (43 per 100,000), whilst amongst women the highest age-specific cumulative incidence is seen at 25 per 100,000 in the age group of 85 years and older. The median age of death for men in 2011 was 77 years old, five years after the average age of onset, while for women it was 82 years, or seven years after the average age of onset.

About 1,000 men and 850 women die each year from CLL in Germany. The number of cases (Figure 1 ) as well as the age-standardised mortality rates (Figure 2 ) have remained for the most part constant over the last several years.

Figure 1: Estimated number of new cases and number of registered deaths due to CLL in Germany

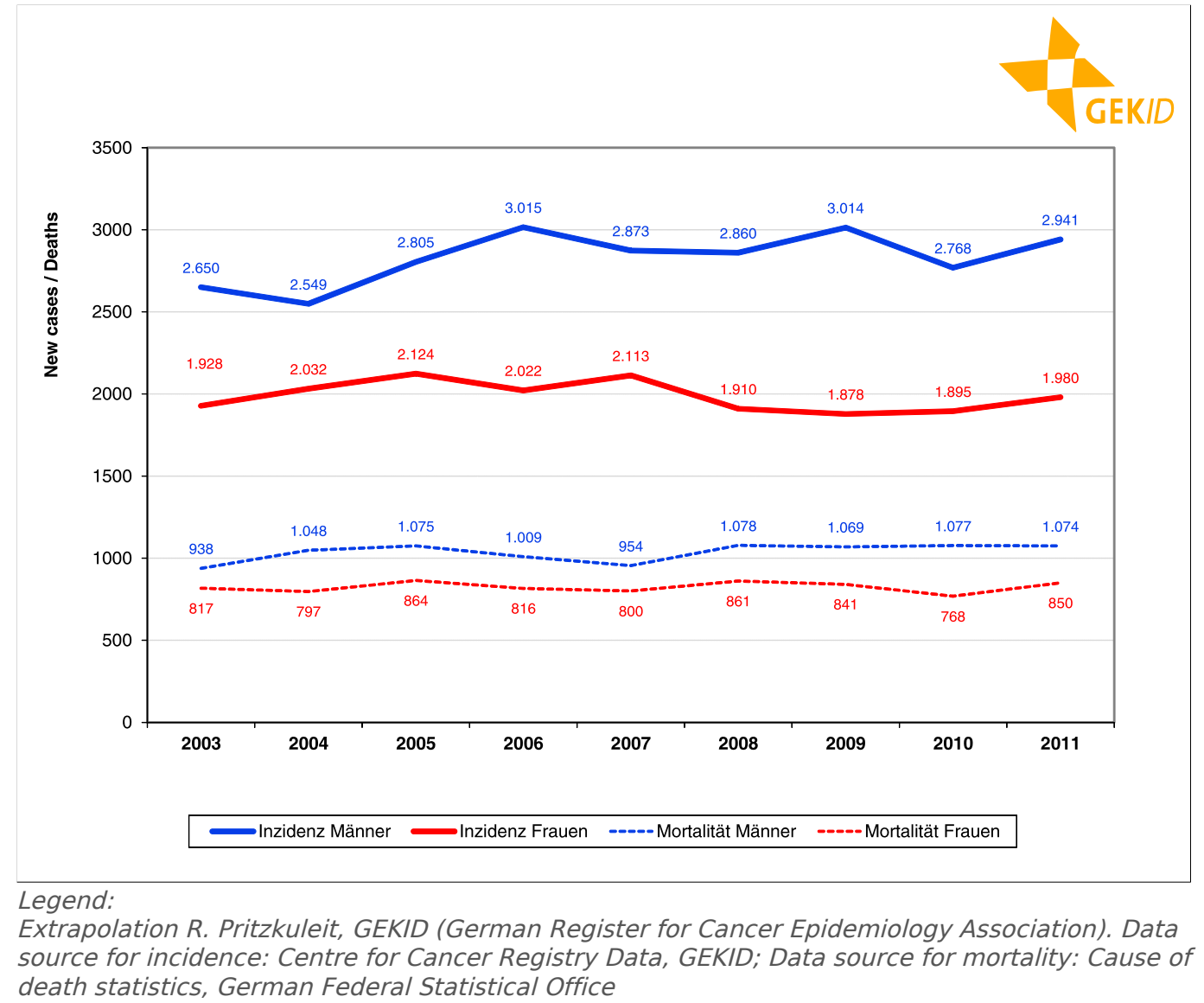


Figure 2: Age-adjusted incidence rate and estimated age-adjusted mortality rate from CLL in Germany

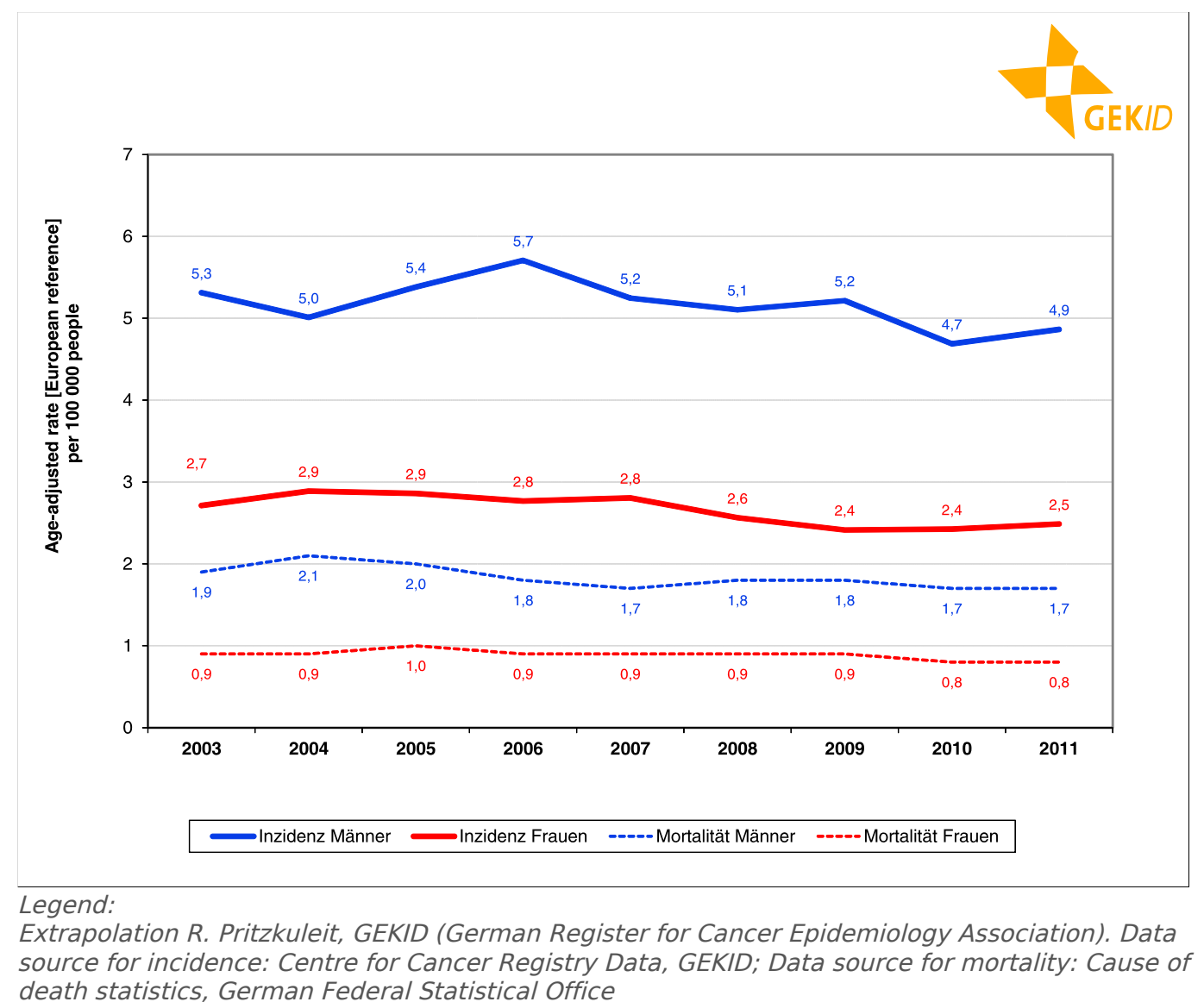


Figure 3: Age-specific incidence of CLL in Germany (extrapolation)

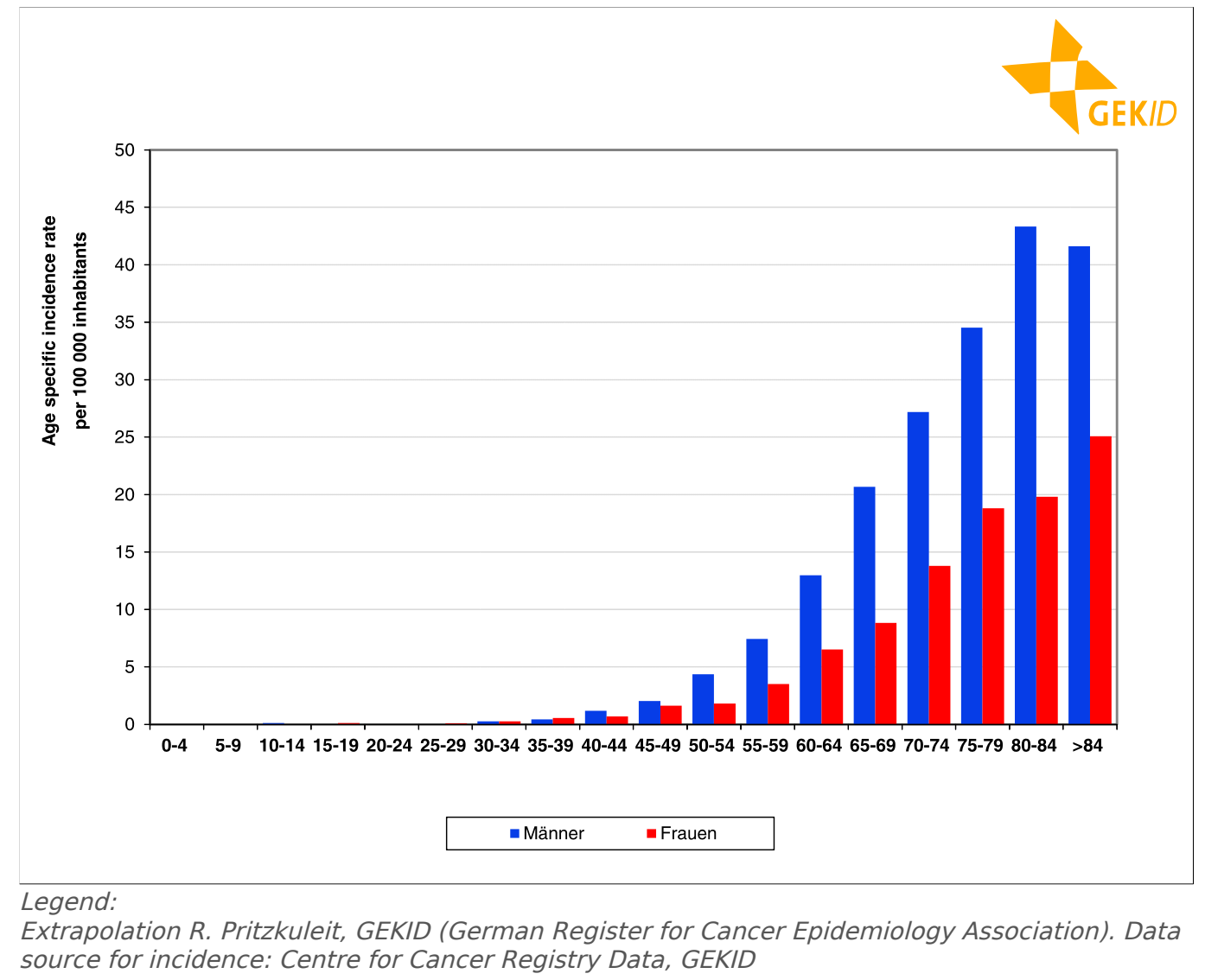

\subsection{Pathogenesis}

Although Chronic Lymphocytic Leukemia has a characteristic immunophenotype, it is biologically and clinically heterogeneous. It arises from antigen-exposed, mature B cells. The key elements of its pathogenesis are the inhibition of apoptosis and the dysregulation of cell proliferation. Genome analysis has resulted in the identification of numerous genetic aberrations and increasingly to a recognition of clinically relevant subgroups with different signal transduction pathways [3].

Of particular importance is the B-cell receptor-signalling pathway, since the blockade of critical kinases, including PI3K and BTK, can be exploited therapeutically. Even BCL-2-dependent signalling pathways play a pathogenetic role in the apoptosis defect seen with CLL, whereby $\mathrm{BH} 3$ mimetics can reverse this effect through their pro-apoptotic effects, and for this reason have already been tested therapeutically in clinical studies.

The growth and circulation of CLL cells is determined by interactions with the lymphatic microenvironment [4]. 


\subsection{Risk Factors}

The risk of developing CLL is increased by the following factors:

- Acquired

- Organic solvents, e.g. benzene according to the German occupational diseases ordinance (no. 1318) [5]

- Hereditary

- First-degree relatives of CLL patients have an 8.5-fold increased risk of developing CLL and a 2.6-fold increased risk of developing another indolent lymphoma [6]. Due to the low incidence of lymphatic neoplasias, the absolute risk of getting the disease amongst family members is still low.

- A small group of patients originates from families with a strikingly high incidence of CLL and other indolent lymphomas [6]. The genetic basis of this predisposition is still unclear.

CLL is generally preceded by an undiagnosed, clinically asymptomatic preliminary stage involving a proliferation of clonal B cells. These cells have the biological characteristics of leukemia cells and this condition is referred to as a monoclonal B lymphocytosis (MBL); see Onkopedia Monoclonal B Lymphocytosis. MBL is detectable in more than $5 \%$ of people aged over 60 . The risk of transformation into a CLL requiring treatment is approximately $1 \%$ / year [7].

\section{Prevention and screening}

\subsection{Screening}

There is no evidence for any effective means of preventive treatment. Screening based on the identification of a monoclonal B lymphocytosis has not yet been established. It would only be useful if an early diagnosis of CLL were to lead to a significant improvement in prognosis.

\section{Clinical picture}

The disease is characterised by a blood lymphocytosis, often detected incidentally. With progression of the disease, lymphadenopathy, splenomegaly and hepatomegaly, signs of bone marrow failure and sometimes even autoimmune cytopenias can occur. Clinical symptoms can also manifest themselves as B symptoms and an increased susceptibility to infections. 


\section{Diagnosis}

\subsection{Diagnostic criteria}

According to the criteria of the International Workshop on CLL (IWCLL) 2008 a diagnosis of CLL is defined by the following criteria [8]:

- Detection of clonal B-lymphocytes at a count of $\geq 5,000$ per microlitre in the peripheral blood. Below this value, monoclonal B-lymphocytosis ( $\mathrm{MBL}$ ) can be diagnosed when no symptoms (B symptoms, lymphadenopathy, hepatomegaly, splenomegaly, cytopenia etc.) are present.

- Predominance of small, morphologically mature lymphocytes upon cytological examination of the blood smear.

- Co-expression of the B cell antigens CD19, CD20, CD23 and the T cell antigen CD5 upon multiparametric immunophenotyping. Other characteristics are the relatively weak expressions of surface immunoglobulin, CD20 and CD79b. The monoclonality of the cells can be assessed by light chain restriction (Ig or Ig), preferably by double labelling of CD19/kappa CD19/ lambda.

Characteristic findings from microscopy, immunophenotyping and genetics are published in the Chronic Lymphocytic Leukemia data base at https:// www.onkopedia.com/de/wissensdatenbank/wissensdatenbank/chronischelymphatische-leukaemie-cll.

\subsection{Diagnostics}

The diagnostic procedure depends on the primary set of findings, usually characterised by the main finding of lymphocytosis with or without concomitant lymphadenopathy. If a patient is suspected of having CLL, the following tests are recommended (see Tables 1 and 2).

Table 1: Diagnosis of suspected CLL

\begin{tabular}{|l|l|}
\hline Diagnostics & Comments \\
\hline Case history & $\begin{array}{l}\text { General weakness, B-symptoms, susceptibility to infections, etc., } \\
\text { earlier blood counts / leukocyte counts, family history }\end{array}$ \\
\hline Physical examination & $\begin{array}{l}\text { Lymph node status, organomegaly, } \\
\text { signs of bleeding disorders or anemia }\end{array}$ \\
\hline Blood count & $\begin{array}{l}\text { Leukocytes with differential blood counts (microscopic differentiation), } \\
\text { platelets, hemoglobin, reticulocytes (with signs of anemia) }\end{array}$ \\
\hline Multiparametric \\
immunophenotyping & $\begin{array}{l}\text { - Expression of CD19 and CD23 } \\
\text { - Woexpression of CD5 } \\
\text { - Monoclonality of IgKappa or IgLambda }\end{array}$ \\
\hline Bone marrow & $\begin{array}{l}\text { Not usually necessary for diagnosis, but may be indicated during disease pro- } \\
\text { gression to clarify unclear cytopenias or the quality of remission }\end{array}$ \\
\hline
\end{tabular}




\begin{tabular}{|l|l|}
\hline Diagnostics & Comments \\
\hline Lymph node biopsy & $\begin{array}{l}\text { Only in the absence of leukemic washout or if transformation into an aggressive } \\
\text { lymphoma is suspected (Richter's syndrome) }\end{array}$ \\
\hline
\end{tabular}

Table 2: Additional diagnostics before initiation of a therapy

\begin{tabular}{|l|l|}
\hline Diagnostics & Comments \\
\hline Genetics & $\begin{array}{l}\text { - del(17p13) * } \\
\text { - TP53 mutation analysis } \\
\text { - further genetic studies if an atypical phenotype is present to distinguish it from other } \\
\text { indolent lymphomas }\end{array}$ \\
\hline $\begin{array}{l}\text { Further laboratory } \\
\text { analysis }\end{array}$ & $\begin{array}{l}\text { Depending on symptoms and planned treatment, e.g. } \\
\text { Haptoglobin and Coombs test in cases of suspected hemolysis, and prior to the initia- } \\
\text { tion of a fludarabine- containing regimen } \\
\text { - GFR with a planned fludarabine therapy } \\
\text { Quantitative determination of immunoglobulins where immunodeficiency is suspected } \\
\text { CMV status (serology) before initiating an alemtuzumab- } \\
\text { containing therapy }\end{array}$ \\
\hline Ultrasonography & Abdomen: Spleen, liver, lymph nodes \\
\hline
\end{tabular}

Legend:

* The data for the poor prognosis of patients with deletion $17 p 13$ is based on molecular cytogenetic analysis via FISH. The group of patients with inactivation of p53 by mutation overlaps largely with the group of patients showing a del17p13, but is not identical.

\subsection{Classification (staging)}

For staging according to Binet (more commonly used one in Europe, see Table 3) [9] or Rai [10], only a physical examination and a blood count analysis are necessary. The results of imaging (organomegaly in sonography, CT) are not relevant for the staging process.

Table 3: Staging according to Binet [4]

\begin{tabular}{|c|l|c|}
\hline Stage & Definition & Median survival \\
\hline A & $\begin{array}{l}\text { Hemoglobin } \geq 10 \mathrm{~g} / \mathrm{dl} \\
\text { Platelets } \geq 100,000 / \mu \mathrm{l} \\
<3 \text { affected regions }{ }^{2}\left(\mathrm{LN}^{1}, \text { liver or spleen }\right)\end{array}$ & $>10$ years \\
\hline B & $\begin{array}{l}\text { Hemoglobin } \geq 10 \mathrm{~g} / \mathrm{dl} \\
\text { Platelets } \geq 100,000 / \mu \mathrm{l} \\
\geq 3 \text { affected regions }{ }^{2}\left(\mathrm{LN}^{1}, \text { liver or spleen }\right)\end{array}$ & 5 years \\
\hline C & $\begin{array}{l}\text { Hemoglobin }<10 \mathrm{~g} / \mathrm{dl} \\
\text { Platelets }<100,000 / \mu l\end{array}$ & $2-3$ years \\
\hline
\end{tabular}

Legend:

${ }^{1}$ LN = lymph nodes;

2 the regions ( $n=5$ ) include cervical, axillary or inguinal $L N$ enlargements (unilateral or bilateral), or liver and spleen enlargements (detection only upon physical examination).

\subsection{Prognostic factors}

Biological prognostic factors, beyond the molecular cytogenetics mentioned in chapter 5.2 (see Table 2), such as thymidine kinase, beta-2-microglobulin, mutational status of the variable segments of the immunoglobulin heavy chain genes 
(IGHV), SF3B1/NOTCH1 mutations and other genomic aberrations, and flow cytometric parameters such as CD38 or ZAP70 expression require further prospective validation and do not currently form the basis of specific, therapeutic considerations outside of clinical trials. Their routine analysis is not indicated outside of clinical trials.

\subsection{Differential diagnosis}

The most common differential diagnoses are:

- Monoclonal B lymphocytosis

- Reactive lymphocytosis (viral infections, connective tissue diseases)

- Other lymphomas with leukemic course (for follicular lymphoma see Onkopedia Follicular lymphoma, lymphoplasmacytic lymphoma, for marginal zone lymphoma see Onkopedia Marginal zone lymphoma, for mantle cell lymphoma see Onkopedia Mantle cell lymphoma, B-prolymphocytic leukemia (B-PLL)).

- Hairy cell leukemia, see Onkopedia Hairy cell leukemia

Special attention is needed for differentiation from mantle cell lymphoma due to its similar immunophenotype with coexpression of CD19 and CD5, whereas mantle cell lymphoma, in contrast to CLL, is usually negative for CD23. For a nonclassical immunophenotype of CLL, either a FISH analysis is indicated to exclude the typical aberration of a mantle cell lymphoma (i.e. the translocation $11 ; 14$ ), or a histological examination of the excised lymph nodes to exclude overexpression of CyclinD1.

\section{Therapy}

\subsection{Therapy structure}

CLL is not currently curable by conventional chemotherapy or antibody-based therapies. The only curative option is allogeneic stem cell transplantation.

An indication for therapy generally arises in Binet stage $C$ or indeed in Binet stage $B$ or $A$ if other criteria requiring treatment are fulfilled:

- Appearance / exacerbation of anemia / thrombocytopenia

- Massive (> $6 \mathrm{~cm}$ below the costal margin), progressive or symptomatic splenomegaly; Note: the spleen size varies between individuals depending on height and weight

- Massive (> $10 \mathrm{~cm}$ in diameter), progressive or symptomatic lymphadenopathy

- Lymphocyte doubling time of less than 6 months, or a $50 \%$ increase in 2 months, starting from a base level of at least 30,000 lymphocytes / $\mu \mathrm{l}$, and after exclusion of other causes of a lymphocytosis 
- Autoimmune cytopenia which is refractory to a standard therapy

- One of the following constitutional symptoms

- Unintentional weight loss $>10 \%$ in 6 months

- Fever of unknown origin for more than 2 weeks

- Night sweats for more than one month without evidence of infection

- Severe fatigue

The structure of the therapy is summarised in Figure 4.

Figure 4: First-line treatment of CLL

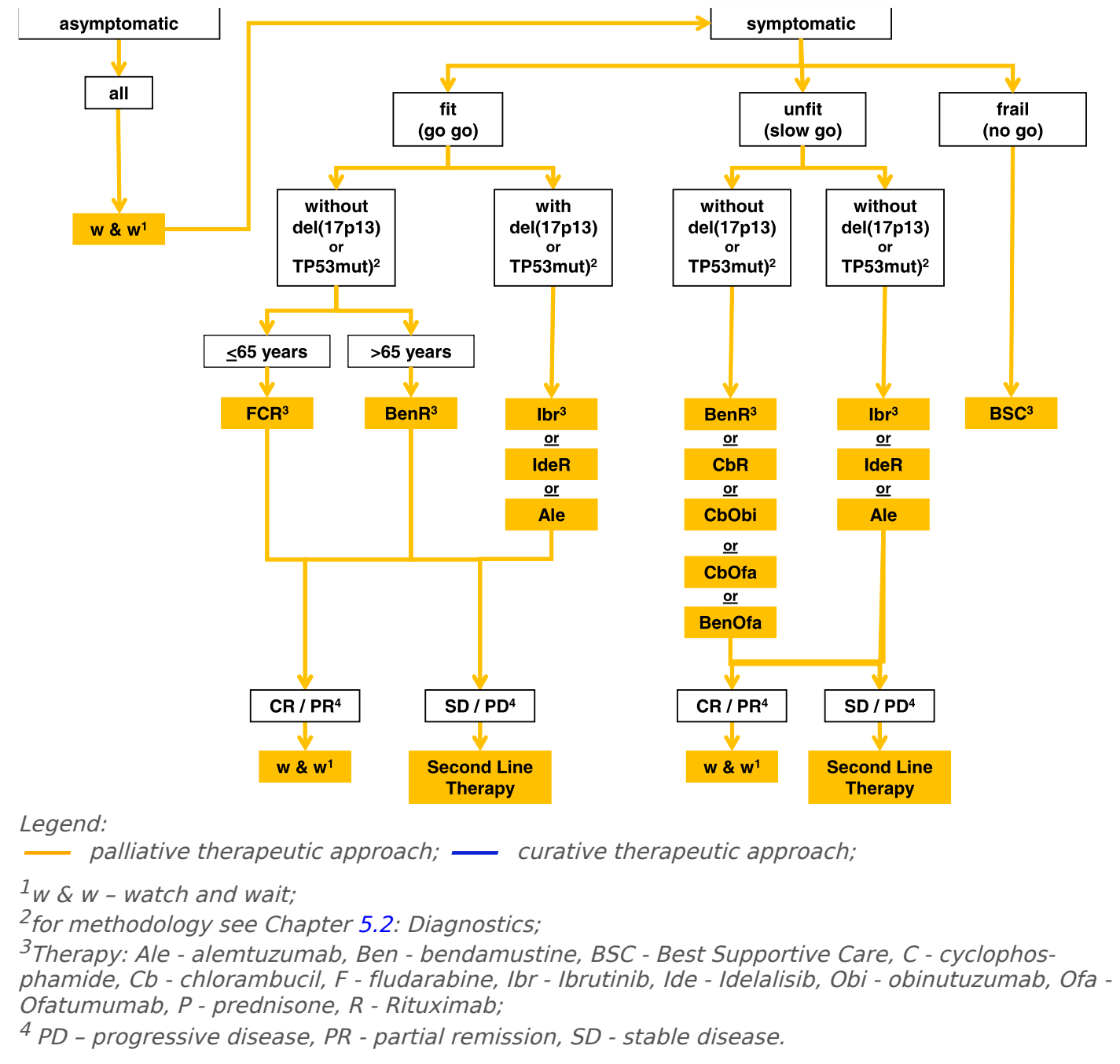

\subsubsection{First-line therapy}

The choice of treatment for CLL depends on the presence of any comorbidities (determined e.g. by the CIRS / Cumulative Illness Rating Scale score), genetic status, renal function and to a lesser extent patient age. Wherever possible, the therapy should take place within the framework of a clinical trial. 


\subsubsection{Patients without del(17p13) or TP53 mutation}

\subsection{Fit ("go go")}

For physically fit patients (e.g. a CIRS $<6$ ) with normal renal function and lack of clinically relevant comorbidity, the first-line treatment of choice outside of clinical trials consists of a combination therapy with fludarabine, cyclophosphamide and rituximab (FCR) [11], see Chronic Lymphocytic Leukemia - Therapy protocols.

An alternative to FCR can be the combination of bendamustine with rituximab $(B R)$, especially where there is an uncontrolled autoimmune hemolytic anemia or renal impairment. Based on the results of one study, in which BR was examined in a randomised comparison with FCR as a first-line therapy (CLL10 protocol of the GCLLSG), BR was recommended in preference to FCR in fit patients beyond their 65th birthday due to its more favourable toxicity profile. In younger, physically fit patients (up to 65 years), FCR remains the first choice for a first-line therapy, see Chronic Lymphocytic Leukemia - Study results.

\subsection{Unfit ("slow go")}

For patients with impaired renal function and/or increased comorbidity, there are at least two options for chemotherapy, namely chlorambucil or bendamustine. In direct comparisons, bendamustine has been proven to be superior to chlorambucil in terms of remission rate, the rate of complete remissions and progressionfree survival [13]. In a Phase III study (CLL11 protocol of the GCLLSG) it was shown that the addition of rituximab or obinutuzumab improved response rate and progression-free survival compared to a chlorambucil monotherapy [14]. Combining obinutuzumab and chlorambucil also improved overall survival compared to chlorambucil alone, but compared to rituximab and chlorambucil there was no significant difference regarding overall survival time, see Chronic Lymphocytic Leukemia - Study results.

The results of a randomised comparison between chlorambucil / rituximab and bendamustine / rituximab (MaBLe Trial) have not been made available yet.

In another Phase III trial in patients unsuitable for fludarabine therapy, ofatumumab in combination with chlorambucil achieved higher remission rates and longer progression-free survival than monotherapy with chlorambucil; see Chronic Lymphocytic Leukemia - study results. On the basis of additional Phase II study data, ofatumumab has received approval as a first line therapy in combination with chlorambucil or bendamustine.

In summary, comorbid patients as well should receive treatment with bendamustine (dose reduced to $70 \mathrm{mg} / \mathrm{m}^{2}$, day $1+2$ ) in combination with rituximab or ofatumumab, or with chlorambucil in combination with rituximab and ofatumumab or obinutuzumab. 
Monotherapy with fludarabine at a standard dosage is not recommended for comorbid patients due to its increased toxicity compared to a chlorambucil or bendamustine-based therapy [15].

Standard recommendations for the doses of chemotherapy or immunotherapy are based on the specifications of multicentre studies. In elderly and comorbid patients with CLL, a dose reduction may be required, sometimes with the first dose, but more often during the course of further therapeutic cycles as an adaptation towards individual sensitivities.

\subsection{Frail ("no go")}

For patients with poor general health, supportive therapy is paramount. This may also include administration of antineoplastic drugs such as steroids, chlorambucil, bendamustine or rituximab at adapted doses.

\subsubsection{Patients with a confirmed del(17p13) or TP53 mutation}

Patients with a confirmed del(17p13) or a TP53 mutation have a lower response rate and shorter progression-free/overall survival after chemotherapy and chemoimmunotherapy (chlorambucil, fludarabine-containing regimens, bendamustine, also in combination with rituximab). In patients without relevant comorbidity but with CLL requiring therapy and del(17p13) /TP53 mutation there are alternative approaches available for first-line therapy such as ibrutinib, idelasilib/rituximab or alemtuzumab. The indication for allogeneic blood stem cell transplantation, which was previously recommended as a standard first-line therapy in these patients [16], should be given critical consideration after weighing up of individual risks considering age, comorbidities, and the availability of perfectly matched donors [17]. Conceptually, allogeneic blood stem cell transplantation might receive consideration as a rescue procedure for providing consolidation where only an initial response is seen or where disease is initially refractory to therapy. If circumstances permit, such patients should be treated within a clinical trial setting.

\subsubsection{Second-line therapy}

The choice of therapy after relapse depends on a number of individual factors. In addition to age and comorbidities of the patient, these include first and foremost clinical parameters such as the type of primary treatment and the duration of remission achieved. The algorithm is illustrated in Figure 5. If circumstances permit, such patients should be treated within a clinical trial setting. 
Figure 5: Second-line treatment of CLL

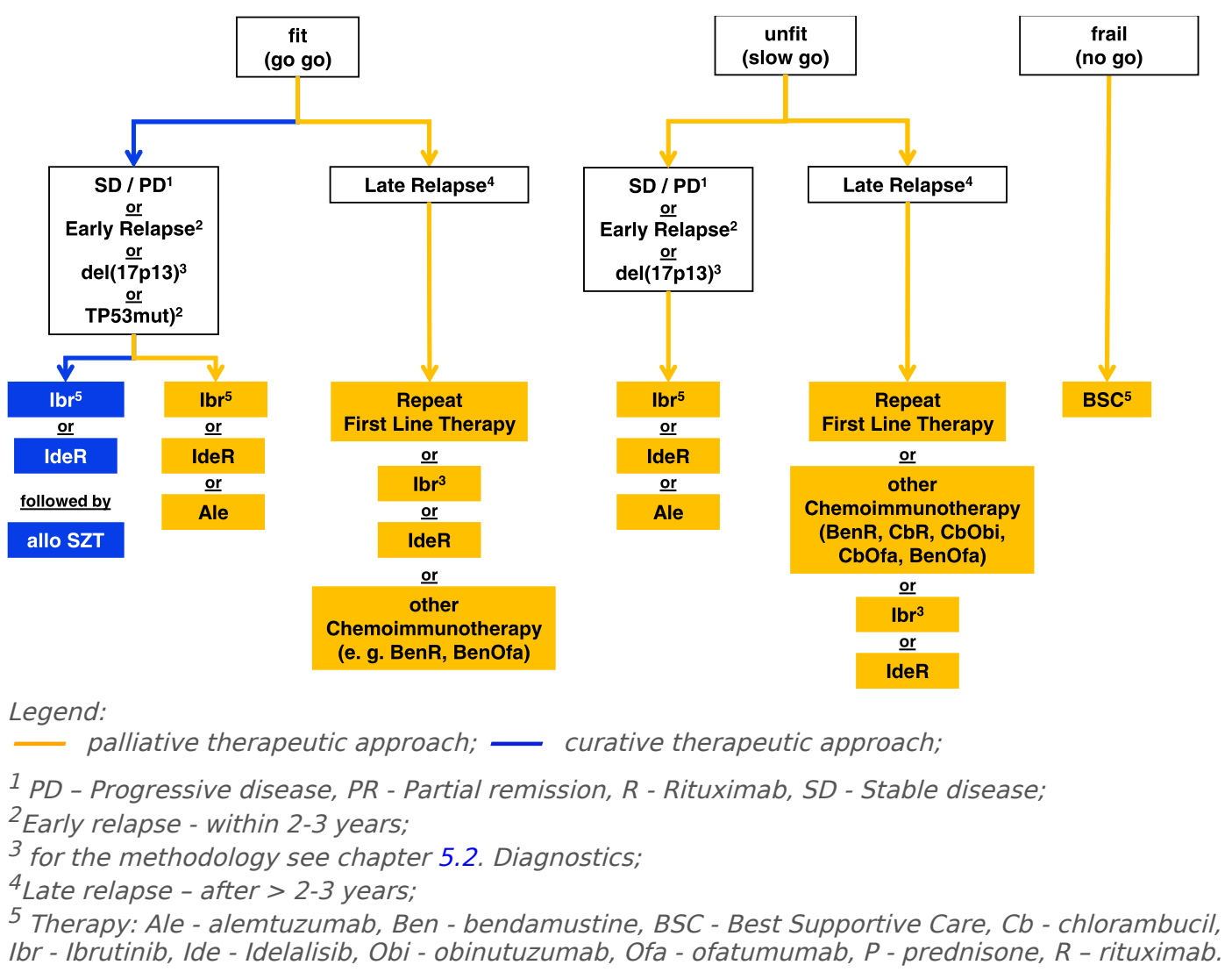

\subsubsection{Progressive disease / therapy resistance / early relapse}

Patients who fail to respond to current standard therapies (FCR, BenR, CBR CbObi, CbOfa, BenOfa) or who undergo an early relapse ( $<2$ years), or relapsed patients with a confirmed del(17p13) or a TP53 mutation have a poor prognosis. They are also referred to as high-risk CLL (high-risk CLL, highest risk CLL, ultrahigh risk CLL) patients [17]. Their median overall survival time is just 1 - 2 years from the date of the initiation of salvage therapy.

One option is the pan-lymphocyte antibody alemtuzumab [18]. Formally its use is strictly off-label in Germany due to the fact that MabCampath (alemtuzumab) has been withdrawn from the market. Upon treatment with alemtuzumab, an extended anti-infective prophylaxis and close infectiological monitoring are required. With a positive CMV serology, CMV reactivations must be reckoned with.

In patients with an aggressive course of CLL (Richter's transformation), chemoimmunotherapy based on R-CHOP can be performed in the same way as is carried out for non-Hodgkin's lymphoma.

Currently two new substances have been approved alone or in combination with antibodies for relapsed/therapy refractory CLL. Compared with ofatumumab, the BTK inhibitor Ibrutinib led to a significant improvement in progression-free survival and overall survival [19]; see Chronic Lymphocytic Leukemia - Study results. Also for the PI3K inhibitor Idelalisib in combination with rituximab, there is 
relevant Phase III data with evidence of a survival benefit (PFS/OS) over rituximab monotherapy alone [20].

The monoclonal anti-CD20 antibody ofatumumab is approved for patients who have been refractory to a fludarabine-containing schema and alemtuzumab (in Germany and Austria), and/or for those not suited to receive these therapies (in Switzerland). However, ofatumumab was inferior to ibrutinib therapy, so in cases of relapse/therapy resistance, this antibody should only be used when an ibrutinib therapy is unsuccessful, although for this sequence no reliable data currently exists for ofatumumab.

\subsubsection{Late relapse}

Despite limited data, it seems justified in principle, where there is a good response and a longer duration of remission of at least 2-3 years (depending on the intensity of the therapy), to use the same regimen again as was used for the first-line therapy. As a matter of principle it can be assumed that with addition of rituximab, an increase in effectiveness can be expected. In addition to intensive fludarabine-containing combination therapies, such as FCR combination therapy, other available relapse therapies include bendamustine/rituximab [21], ibrutinib and idelalisib/rituximab.

\subsubsection{Allogeneic stem cell transplantation}

For patients with therapy-refractory, early relapsed CLL or confirmation of a del(17p13) or TP53 mutation (high-risk CLL, see chapter 6.1.2.1), allogeneic stem cell transplantation represents a reasonable option, as long as the patient's history and physical condition permit it [22]. Another indication in relapse is the demonstration of a del(17p13) or TP53 mutation with a disease requiring therapy. Patients with Richter's transformation should be offered an allogeneic transplantation, provided that the fitness of the patient and the donor situation allows this. The value of allogeneic transplantation as a consolidation measure after initial relapse following targeted medication such as ibrutinib and idelalisib/rituximab is currently a matter of discussion and is not generally recommended outside of clinical trials.

The indication, timing and implementation of a transplantation (including the search for a donor) should be clarified in close cooperation with a transplant centre before initiating a salvage or first-line therapy. An important prognostic factor for achieving a long-term control of the disease is the presence of a relapse at the time of allogeneic transplantation. Wherever possible, transplantation should only be carried out within the framework of clinical trials (e.g. CLL X4 protocol of the GCLLSG).

\subsubsection{Autologous stem cell transplantation}

High-dose therapy with autologous blood stem transplantation, in comparison with chemoimmunotherapy, leads to no increase in remission or survival rates, so 
this procedure can no longer be recommended for the treatment of relapsed/ refractory CLL.

\subsubsection{Supportive therapy and treatment of complications}

Often during the course of disease, CLL patients show infectious disease complications which are exacerbated by the decrease in immunoglobulin concentrations and other mechanisms associated with an acquired immune deficiency. Particularly careful monitoring, e.g. for chronic or recurrent bronchitis, is indicated. Prophylactic substitution with immunoglobulins reduces the risk of serious infections, but has no significant effect on mortality [23]. These can be carried out in patients with hypogammaglobulinemia and a simultaneously increased infection rate.

Age appropriate vaccinations (e.g. against influenza or pneumococci) are recommended, although the formation of specific antibodies towards these vaccines may be hindered in such cases. Travel vaccinations should only be performed after consultation with an appropriate medical specialist, since amongst other possible scenarios live vaccines might endanger the life or well-being of the patient.

\subsection{Therapeutic modalities}

\subsubsection{Drug tumour therapy - substances}

The results of randomised clinical trials of the individual substances and combinations are summarised in Chronic Lymphocytic Leukemia - Study results. Information regarding the approval status of the drugs suitable for the treatment of CLL is given in Chronic Lymphocytic Leukemia - Approval status for Germany, Austria and Switzerland.

\subsubsection{Alemtuzumab}

Alemtuzumab is a humanised, monoclonal antibody directed towards the CD52 antigen present on lymphocytes and monocytes. Alemtuzumab is effective as a first-line and second-line therapy for patients with CLL. In the first-line treatment of unselected patients, alemtuzumab compared to chlorambucil achieved with $83 \%$ vs $55 \%$ a significantly higher remission rate and a significant prolongation of progression-free survival, but no significant prolongation of median survival, see Chronic Lymphocytic Leukemia - Study results. Alemtuzumab has a higher efficacy especially amongst patients with inactivation of p53 caused by a del(17p13) or by mutation. Due to the increased rate of side effects, alemtuzumab is only recommended for first-line treatment in patients with inactivation of p53 and for refractory patients. Alemtuzumab is used as a single agent and in combination therapies. Side effects of alemtuzumab monotherapy of CTCAE grade 3/4 include fever $(8 \%)$, reactivation of CMV (4\%), symptomatic CMV infection (4\%) and chills (3\%). Initially, intolerance reactions can occur, often with urticaria, hypotension 
and cardiac complications. Alemtuzumab is administered intravenously or subcutaneously [18].

In August 2012, the pharmaceutical company authorised to produce Alemtuzumab withdrew the approval for alemtuzumab in patients with CLL for commercial reasons. Since then the cost-free import of MabCampath from the US has been organised by a British company.

\subsubsection{Bendamustine}

Bendamustine is a nitrogen mustard derivative. It is an alkylating agent and has the same properties as a purine antimetabolite. As a monotherapy for previously untreated patients, remission rates were $68 \%$ and progression-free survival was 21.8 months and significantly higher than a comparator monotherapy with chlorambucil (31\%, 8.0 months); see Chronic Lymphocytic Leukemia - Study results. Bendamustine is used in CLL as a single agent or in combination with rituximab $[13,21]$. Side effects of CTCAE grade $3 / 4$ are related to hematopoiesis: neutropenia $(23 \%)$, thrombocytopenia (12\%), anemia (2.5\%). When these occur, dose adjustments are required. Other frequent side effects include fever, nausea/ vomiting and skin exanthema. Bendamustine is applied intravenously.

\subsubsection{Chlorambucil}

Chlorambucil is an alkylating agent. It has been used for more than 50 years for the treatment of CLL. The combination with prednisone for 3 days was used for decades as a less aggressive form of chemotherapy (known as the "Knospe Schema"), but is no longer recommended. The now conventional chlorambucil monotherapy $(0.4 \mathrm{mg} / \mathrm{kg}$ BW every 14 days with dose escalation to $0.1 \mathrm{mg} / \mathrm{kg} \mathrm{BW}$ per cycle up to a maximum dose of $0.8 \mathrm{mg} / \mathrm{kg} \mathrm{BW}$ ) has served as the standard comparative therapy for testing the added benefits of newer substances. Chlorambucil is well tolerated. The side effects are dose-dependent. They are primarily hematological and manifest themselves as neutropenia, thrombocytopenia and anemia. Side effects of CTCAE grade 3/4 are rare and can be avoided through dose reduction. Chlorambucil is administered orally.

\subsubsection{Cyclophosphamide}

Cyclophosphamide has an effect as a monotherapy in CLL, but is usually used in combination with fludarabine and rituximab (FCR). The main side effect of cyclophosphamide is its hematotoxicity. At higher doses (> $1000 \mathrm{mg}$ ) a hemorrhagic cystitis can occur which is preventable by prophylactically administering uromitexan. Cyclophosphamide is usually administered intravenously. Cyclophosphamide is also effective in the treatment of autoimmune diseases. 


\subsubsection{Fludarabine}

Fludarabine is a purine analogue. It was first synthesised in the late 1960s and has been used to treat CLL since the 1980s. Compared to chlorambucil or alkylating agent-containing combination therapies, monotherapy leads to an increase in remission rates, but not overall survival; see Chronic Lymphocytic Leukemia Study results. Compared to $\mathrm{CHOP}$, the efficacy results were not significantly different, but tolerability was significantly better. Side effects of fludarabine monotherapy of CTCAE grade $3 / 4$, occurring in more than $5 \%$ of patients, include neutropenia, anemia, thrombocytopenia and infections. A critical side effect of fludarabine monotherapy is the increased rate of autoimmune cytopenias or even pure red cell aplasia.

Fludarabine is now recommended as a standard therapy in combination with cyclophosphamide and rituximab (FCR) [11]. Fludarabine is effective via intravenous, subcutaneous or oral administration. In the recommended therapeutic regimens, it is administered intravenously.

\subsubsection{Ibrutinib}

Ibrutinib is an inhibitor of Bruton's tyrosine kinase (BTK). This kinase plays a key role in the development, differentiation, signalling and survival of B lymphocytes. In the pivotal study on patients with relapsed or refractory CLL, ibrutinib resulted in a remission rate of $71 \%$ and a progression-free survival at 26 months of $75 \%$ [24]. The most common side effects were diarrhoea, fatigue, anemia and infections of the upper airways. Serious side effects of CTCAE grade 3/4 were pneumonia (12\%) and dehydration (6\%). Therapy with Ibrutinib leads initially to a lymphocytosis due to a washout of leukemic cells from lymphatic compartments. In a randomised study in patients who had already received first-line therapy, ibrutinib was significantly superior to ofatumumab [19]. Although the lymphocytosis may persist for several months, it is not correlated with the treatment response. Ibrutinib is administered orally.

\subsubsection{Idelalisib}

Idelalisib is a selective inhibitor of the delta isoform of phospho-phosphatidylinositol-3-kinase (PI3Kס). This kinase plays an important role in B-cell receptor-induced signalling in mature B lymphocytes and in the pathogenesis of CLL. In the pivotal study on patients with relapsed CLL, the combination of idelalisib/rituximab achieved a remission rate of $81 \%$ compared to just $13 \%$ for rituximab monotherapy [20]. Both the progression-free survival and the overall survival at 12 months were significantly prolonged. Serious side effects for the combination therapy of CTCAE grade $3 / 4$ included neutropenia (34\%), thrombocytopenia (10\%), anemia $(5 \%)$, increase in transaminases (5\%), diarrhoea $(4 \%)$, fever (3\%), fatigue $(3 \%)$ and chills ( $2 \%)$. Idelalisib is administered orally. 


\subsubsection{Obinutuzumab}

Obinutuzumab is an anti-CD20 antibody with an altered glycosylation pattern. In treatment-naïve, comorbid patients, treatment in combination with chlorambucil led to a significant improvement in remission rates of $77.3 \%$ vs $31.4 \%$, and a significant prolongation of progression-free (26.7 vs 11.1 months) and overall survival compared to chlorambucil monotherapy [14]; see Chronic Lymphocytic Leukemia - Study results. Serious side effects of combination therapy with chlorambucil in CTCAE grade $3 / 4$ included infusion-related reactions $(21 \%)$, neutropenia (35\%), thrombocytopenia (11\%), thrombocytopenia (11\%) and anemia (5\%). Obinutuzumab is administered intravenously.

\subsubsection{Ofatumumab}

Ofatumumab is an anti-CD20 antibody with a different epitope-binding site, and higher in vitro activity than rituximab. The approval was based on a phase II study in patients undergoing treatment with fludarabine and alemtuzumab. In that study, about half of the patients achieved remission with a median progression-free survival time of 5.5-6 months. Serious side effects in CTCAE grade 3/4 included neutropenia (8\%) and infections (9\%). Infusion-related reactions of grade $1 / 2$ occurred in about $60 \%$ of patients. In the first-line treatment of patients with contraindications to fludarabine, a combination of ofatumumab with chlorambucil led to a significantly greater increase in remission rate, the rate of complete remissions, and progression-free survival time compared to chlorambucil monotherapy; see Chronic Lymphocytic Leukemia - Study results. Serious side effects in CTCAE grade $3 / 4$ include neutropenia (26\%), infections (15\%) and infusion-related reactions (10\%). Ofatumumab is administered intravenously and is approved for first-line therapy in combination with bendamustine.

\subsubsection{Prednisone / prednisolone}

Prednisone or prednisolone is the most commonly used glucocorticoid for CLL. For decades it was an integral part of the therapeutic standard in Germany in combination with chlorambucil (known as the "Knospe Schema"). After randomised trials showed no improvement in survival rates, but a higher rate of complications after addition of prednisone, its use was reduced for the treatment of patients with autoimmune diseases. Short-term side effects of glucocorticoids include flushing, restlessness and disorders in glucose metabolism. Medium and longerterm side effects correspond to the symptoms of Cushing's syndrome, osteoporosis and changes in body shape. Critical side effects especially in CLL patients were infections, especially of a viral or fungal origin, which occurred due to an exacerbation of immunosuppression. Glucocorticoids can be administered orally and intravenously. 


\subsubsection{Rituximab}

Rituximab is a chimeric anti-CD20 antibody. It was first approved for the treatment of patients with indolent lymphoma. Rituximab is effective as a monotherapy for CLL, and its effect is dose-dependent. In combination with fludarabine and cyclophosphamide (FCR), rituximab was the first substance that resulted in a significant prolongation of overall survival [11]; see Chronic Lymphocytic Leukemia - Study results. The most common side effects of rituximab are infusion-related and include fever, chills, nausea and malaise. A cytokine release syndrome can cause severe hypotension with high temperatures, hypoxia and may require intensive care. The incidence of cytokine release syndrome correlates with the tumour mass and depends on the rituximab dosage. Side effects of the FCR combination therapy of CTCAE grade 3/4 include cytokine release syndrome, neutropenia (34\%), infections (25\%), thrombocytopenia (7\%) and anemia (4\%). Rituximab may be administered intravenously and subcutaneously. Only intravenous administration is currently approved for CLL.

\subsubsection{Vincristine}

Vincristine is a vinca alkaloid. In CLL it is a component of combination regimens such as R-CHOP. In addition to causing hematotoxicity, specific side effects include polyneuropathy, starting out as a sensory paresthesia of the extremities. Vincristine is administered intravenously. Intravenous extravasation requires immediate corrective action to be taken.

\subsection{Therapy for autoimmune phenomena}

Autoimmune hemolytic anemia (AIHA) and immune thrombocytopenic purpura (ITP) are common comorbidities with CLL [25]. AlHA is as a rule Coombs-positive. Cold agglutinins with detection of $\lg \mathrm{M}$ antibodies are the exception. Coombsnegative hemolytic anemia has been described after a prior purine analogue therapy. Patients should be advised of the particularly high risk of crises regarding infections. On rare occasions, a pure red cell aplasia (PRCA) may be observed. With isolated occurrence of AIHA or ITP without any other CLL symptoms requiring treatment (see above), treatment with corticosteroids is indicated. Therapeutic options in the absence of response to corticosteroids may include rituximab/ bendamustine, rituximab/cyclophosphamide/dexamethasone (RCD), or possibly $\mathrm{R}-\mathrm{CHOP}$ if patients are physically fit. Monotherapy with purine analogues is contraindicated if active autoimmune phenomena are present.

\section{Rehabilitation}

Patients should be informed early on about the options for outpatient and inpatient rehabilitation and anything else that they may be entitled to according to social and healthcare laws. The wishes of the patient must also be taken into account as regards their preference for a rehabilitation clinic (§ 9 SGB IX). Despite 
this, a recommendation for a clinic specialising in oncology should still be made in order to ensure optimal success for the rehabilitation programme.

\section{Follow-up}

Follow-up for asymptomatic patients should include complete blood count examinations at intervals of 3-6 months, in addition to a clinical examination of the lymph nodes, liver and spleen. Attention should be paid to whether autoimmune cytopenias (AIHA, ITP) or infections have set in. Furthermore, rapid enlargements of the lymph nodes, B symptoms and/or increases in LDH should provide the occasion to exclude (in addition to a relapse of the CLL) any transformation into a highly malignant lymphoma (Richter syndrome).

\section{References}

1. www.gekid.de

2. Dores GM, Anderson WF, Curtis RE, et al. Chronic lymphocytic leukaemia and small lymphocytic lymphoma: overview of the descriptive epidemiology. $\mathrm{Br} J$ Haematol 139:809-819, 2007. DOI:10.1111/j. 1365-2141.2007.06856.x

3. Döhner $H$, Stilgenbauer S, Benner A, et al. Genomic aberrations and survival in chronic lymphocytic leukemia. N Engl J Med 343:1910-1916, 2000. PMID:11136261

4. Burger JA, Gribben JG: The microenvironment in chronic lymphocytic leukemia and other $B$ cell malignancies: insight into disease biology and targeted therapies. Semin Cancer Biol 24:71-81, 2014. DOI:10.1016/ j.semcancer.2013.08.011

5. Berufskrankheitenverordnung: http://www.gesetze-im-internet.de/bkv/ BJNR262300997.html

6. Goldin LR, Landgren O, Marti GE et al.: Familial aspects of chronic lymphocytic leukemia, monoclonal B-cell lymphocytosis (MBL) and related lymphomas. Eur J Clin Med Oncol 2:119-216, 2010. PMID:21191471

7. Rawstron AC, Bennett FL, O'Connor SJ, et al. Monoclonal B-cell lymphocytosis and chronic lymphocytic leukemia (CLL). N Engl J Med 359:575-583, 2008. DOI:10.1056/NEJMoa075290

8. Hallek M, Cheson BD, Catovsky D, et al. Guidelines for the diagnosis and treatment of chronic lymphocytic leukemia: a report from the International Workshop on Chronic Lymphocytic Leukemia updating the National Cancer Institute-Working Group 1996 guidelines. Blood 111:5446-5456, 2008. DOI: 10.1182/blood-2007-06-093906

9. Binet JL, Auquier A, Dighiero $G$ et al. A new prognostic classification of chronic lymphocytic leukemia derived from a multivariate survival analysis. Cancer 48:198-204, 1981. PMID:7237385 
10. Rai KR, Sawitsky A, Cronkite EP, et al. Clinical staging of chronic lymphocytic leukemia. Blood 46:219-234, 1975. PMID:1139039

11. Hallek $M$, Fingerle-Rowson $G$, Fink $A-M$, et al. First-Line Treatment with Fludarabine (F), cyclophosphamide (C), and Rituximab (R) (FCR) Improves Overall Survival (OS) in Previously Untreated Patients (pts) with Advanced Chronic Lymphocytic Leukemia (CLL): Results of a Randomized Phase III Trial On Behalf of An International Group of Investigators and the German CLL Study Group. Lancet 376:1164-1174, 2010. DOI:10.1016/ S0140-6736(10)61381-5

12. Knauf WU, Lissichkov, Aldaoud A, et al. Phase III randomized study of bendamustine compared with chlorambucil in previously untreated patients with chronic lymphocytic leukemia. J Clin Oncol 27:4378-4384, 2009. DOI: $10.1200 / J C O .2008 .20 .8389$

13. Fischer $\mathrm{K}$, Cramer $\mathrm{P}$, Busch $\mathrm{R}$, et al: Bendamustine in combination with rituximab for previously untreated patients with chronic lymphocytic leukemia: a multicenter phase II trial of the German Chronic Lymphocytic Leukemia Study Group. J Clin Oncol 30:3209-3216, 2012. DOI:10.1200/JCO. 2011.39.2688

14. Goede V, Fischer K, Busch R, et al: Obinutuzumab plus chlorambucil in patients with CLL and coexisting conditions. N Engl J Med 370:1101-1110, 2014. DOI:10.1056/NEJMoa1313984

15. Eichhorst BF, Busch R, Stilgenbauer S, et al. No clinical benefit for frontline therapy with fludarabine in comparison to chlorambucil in elderly patients with advanced chronic lymphocytic leukemia. Blood 114:3382-3391, 2009. DOI:10.1182/blood-2009-02-206185

16. Schetelig J, van Biezen A, Brand R, et al: Allogeneic hematopoietic stem-cell transplantation for chronic lymphocytic leukemia with $17 p$ deletion: a retrospective European Group for Blood and Marrow Transplantation analysis. J Clin Oncol 26:5094-5100, 2008. DOI:10.1200/JCO.2008.16.2982

17. Dreger $\mathrm{P}$, Schetelig J, Andersen $\mathrm{N}$ et al: Managing high-risk chronic lymphocytic leukemia during transition to a new treatment era: Stem cell transplantation or novel agents. A position statement of the European Research Initiative on CLL (ERIC) and the European Society for Blood and Marrow Transplantation (EBMT). Submitted.

18. Stilgenbauer $S$, Zenz $T$, Winkler $D$, et al: Subcutaneous alemtuzumab in fludarabine-refractory chronic lymphocytic leukemia: clinical results and prognostic marker analyses from the CLL2H study of the German Chronic Lymphocytic Leukemia Study Group. J Clin Oncol 27:3994-4001, 2009. DOI: 10.1200/JCO.2008.21.1128

19. Byrd JC, Brown JR, O'Brien S, et al: Ibrutinib versus ofatumumab in previously treated chronic lymphoid leukemia. N Engl J Med 371:213-223, 2014. DOI: 10.1056/NEJMoa1400376 
20. Furman RR, Sharman JP, Coutre $S E$, et al: Idelalisib and rituximab in relapsed chronic lymphocytic leukemia. N Engl J Med 370:997-1007, 2014. DOI:10.1056/NEJMoa1315226

21. Fischer $\mathrm{K}$, Cramer $\mathrm{P}$, Busch $\mathrm{R}$, et al: Bendamustine combined with rituximab in patients with relapsed and/ or refractory chronic lymphocytic leukemia: a multicenter phase II trial of the German Chronic Lymphocytic Leukemia Study Group. J Clin Oncol 29:3559-3666, 2011. DOI:10.1200/JCO. 2010.33.8061

22. Dreger $P$, Döhner $H$, Ritgen $M$ et al: Allogeneic stem transplantation provides durable disease control in patients with chronic lymphocytic leukemia: long-term clinical and MRD results of the German CLL Study Group CLL3X trial. Blood 116:2438-2447, 2010. DOI:10.1182/ blood-2010-03-275420

23. Raanani $P$, Gafter-Gvili A, Paul $M$ et al.: Immunoglobulin prophylaxis in hematologic malignancies and hematopoietic stem cell transplantation. Cochrane Database Syst Rev CD006501, 2008. DOI: 10.1002/14651858.CD006501.pub2

24. Byrd JC, Furman RR, Coutre SE, at al: Ibrutinib Targeting BTK with ibrutinib in relapsed chronic lymphocytic leukemia. N Engl J Med. 2013;369:32-42, 2013. DOI:10.1056/NEJMoa1215637

25. Hodgson K, Ferrer G, Pereira A et al.: Autoimmune cytopenia in chronic lymphocytic leukemia: diagnosis and treatment. Brit J Haematol 154:14-22, 2011. DOI:10.1111/j.1365-2141.2011.08707.x

\section{Systemic Therapy - Protocols}

- Chronic Lymphocytic Leukemia - Systemic Therapy - Protocols

\section{Study results}

- Chronic Lymphocytic Leukemia - Study Results (RCT, Metaanalysis)

\section{Links}

\section{Kompetenznetzwerk Maligne Lymphome}

http://www.kompetenznetz-leukaemie.de

Deutsche Leukämie - und Lymphom - Hilfe e. V.

http://www.leukaemie-hilfe.de

\section{Deutsche CLL Studiengruppe}

www.dcllsg.de 


\section{Authors' Affiliations}

Prof. Dr. med. Clemens-Martin Wendtner

Klinikum München-Schwabing

Hämatologie, Onkologie, Immunologie,

Palliativmedizin

Kölner Platz 1

80804 München

Tel: 089 3068-2228

Fax: 089 3068-3912

clemens.wendtner@klinikum-muenchen.de

Prof. Dr. med. Peter Dreger

Universitätsklinikum Heidelberg

Abteilung Innere Medizin $\mathrm{V}$

Im Neuenheimer Feld 410

69120 Heidelberg

Tel: 06221 56-8008

Fax: 06221 56-6824

peter.dreger@med.uni-heidelberg.de

Dr. med. Michael Gregor

Luzerner Kantonsspital

Departement Medizin

Hämatologische Abteilung

$\mathrm{CH}-6000$ Luzern 16

Tel: 0041412055313

michael.gregor@ksl.ch

Prim. Univ.-Prof. Dr. Richard Greil

Landeskrankenhaus Salzburg

Universitätsklinik f. Innere Medizin III

Onkologisches Zentrum

Müllner Hauptstr. 48

A-5020 Salzburg

Tel: $00436624482-2879$

Fax: 0043662 4482-2898

r.greil@salk.at

Prof. Dr. med. Wolfgang Ulrich Knauf

Onkologie-Zentrum Bethanien

Im Prüfling 17-19

60389 Frankfurt

Tel: 069 45-1080

Fax: 069 45-8257

wolfgang.knauf@telemed.de 


\section{Dr. Ron Pritzkuleit}

Institut für Krebsepidemiologie

Krebsregister Schleswig-Holstein

Ratzeburger Allee 160

23538 Lübeck

Tel: 0451 500-5446

ron.pritzkuleit@krebsregister-sh.de

PD Dr. med. Johannes Schetelig

Universitätsklinikum Carl Gustav Carus Dresden

MK1-IST (66c)

Medizinische Klinik I

Fetscherstr. 74

01307 Dresden

Tel: 03514584190

Fax: 03514585362

johannes.schetelig@uniklinikum-dresden.de

Ao. Univ. Prof. Dr. Michael Steurer

Medizinische Universität Innsbruck

Klinik f. Innere Medizin V

Anichstr. 35

A-6020 Innsbruck

Tel: 0043512 504-81386

Fax: 0043512 504-25615

Michael.Steurer@i-med.ac.at

Prof. Dr. med. Stephan Stilgenbauer

Universitätsklinikum Ulm

Klinik für Innere Medizin III

Albert-Einstein-Allee 23

$89081 \mathrm{UIm}$

Tel: 0731 5004-5521

Fax: 0731 5004-5525

stephan.stilgenbauer@uniklinik-ulm.de 